\title{
Comment on Nietner et al. [Virchows Arch (s2012) 461:259-269]
}

\author{
Torsten Goldmann • Ekkehard Vollmer
}

Received: 19 June 2013 / Accepted: 8 July 2013 / Published online: 20 July 2013

(C) Springer-Verlag Berlin Heidelberg 2013

Dear Sirs,

We have read the study of Nietner et al. [Virchows Arch (2012) 461:259-269] with interest. In general, such studies are necessary and new fixatives are needed within the growing demand for immunohistochemical and molecular approaches. The results obtained here with the HOPE technique are not surprising, since the procedure was not done according to the manufacturer's prescriptions (http://www. dcs-diagnostics.de/data/HOPE_Manual_070801.pdf). The authors did the deparaffinization with a 'usual dehydration process', which means an ascending series of ethanol and xylene or another intermedium. This dehydration is not compatible with the HOPE technique since first of all ethanol itself is a fixative, which influences antigenic properties, and, secondly, the HOPE I solution needs to be washed out by acetone in order to achieve a good preservation of the morphology. This explains the poor morphology and the need for pretreatment in immunohistochemistry. The right way to dehydrate HOPE-fixed materials (by acetone) is long known [1], which is why we cannot agree with the procedure used and the results obtained here.

With our best regards

\section{Reference}

1. Olert J, Wiedorn KH, Goldmann T, Kühl H, Mehraein Y, Scherthan H, Niketeghad F, Vollmer E, Müller MA, Müller-Navia J (2001) HOPE fixation: a novel fixing method and paraffin-embedding technique for human soft tissues. Pathol Res Pract 197:823-826

T. Goldmann $(\bowtie) \cdot$ E. Vollmer

Research Center Borstel, Clinical and Experimental Pathology,

Airway Research Center North (ARCN), Member of the German

Center for Lung Research (DZL), Parkallee 3,

23845 Borstel, Germany

e-mail: tgoldmann@fz-borstel.de 